\title{
Trapped interacting Bose gas in nonextensive statistical mechanics
}

\author{
B. Tanatar \\ Department of Physics, Bilkent University, Bilkent, 06533 Ankara, Turkey
}

(Received 8 November 2001; published 18 March 2002)

\begin{abstract}
We study the Bose-Einstein condensation (BEC) phenomenon in an interacting trapped Bose gas using the semiclassical two-fluid model and nonextensive statistical mechanics. The effects of nonextensivity characterized by a parameter are explored by calculating the temperature dependent thermodynamic properties, fraction of condensed particles, and density distributions of condensed and thermal components of the system. It is found that nonextensivity in the underlying statistical mechanics may have large effects on the BEC transition temperature.
\end{abstract}

DOI: 10.1103/PhysRevE.65.046105

PACS number(s): 03.75.Fi, 05.30.Jp, 32.80.Pj

\section{INTRODUCTION}

The nonextensive statistical mechanics introduced by Tsallis [1] is a field of great interest with many applications. In this formulation of statistical mechanics the nonextensive entropy is defined as $S_{q}=\left(k_{B} / q-1\right)\left(1-\Sigma_{i} p_{i}^{q}\right)$ in terms of the probabilities $p_{i}$ for the ensemble to be in the state $i$ and $q$ is the so-called nonextensivity parameter $\left(k_{B}\right.$ is the Boltzmann constant). The basic property of entropy $S_{q}$ is its nonadditivity from which a host of interesting thermodynamic and statistical mechanical results follow. The standard results in Boltzmann-Gibbs statistics are recovered in the limit as $q \rightarrow 1$. The Tsallis approach to statistical mechanics have found various applications ranging from Lévy-like anomalous diffusion [2] to relaxation through electron-phonon interaction [3]. The generalized distribution functions pertinent to classical and quantum systems in this context were studied by a number of authors [4-8].

The successful observation of Bose-Einstein condensation (BEC) in externally confined atomic vapors [9] has also stirred a great deal of interest in the study of interacting boson systems. The ground-state static and dynamic properties and thermodynamics of condensates are extensively investigated and the essential results are compiled in a number of review articles [10]. Experimental results of the temperature dependence of the fraction of atoms in the condensate, the density distribution of condensed and noncondensed atoms provide a testing ground for many-body calculations on these quantum systems.

Recently, Salasnich [11] considered the BEC phenomenon in trapped noninteracting systems within the nonextensive statistical mechanics. He employed an approximate form of the nonextensive $\mathrm{BE}$ distribution function to calculate the BEC transition temperature and other thermodynamic quantities. Fa and Lenzi [12] reexamined the same problem without resorting to approximations to the nonextensive BE distribution function and found considerable differences. The aforementioned works studied noninteracting system of trapped bosons. However, for the current atomic vapors of interest, although being in the dilute gas regime, the interaction effects are very important. Most thermodynamic properties are understood by introducing an effective interaction coupling expressed in term of the $s$-wave scattering length and within a mean-field approach. An attempt to understand interacting Bose gas at zero temperature within the Tsallis statistics has been made by Fa et al. [13].

The purpose of the present paper is to study the effects of nonextensivity on an interacting system of trapped bosons. To this end we use the two-fluid model of interacting bosons at finite temperature developed by Minguzzi et al. [14]. Here the Bose system is considered to be composed of a condensate and a thermal component below the BEC temperature. The noncondensed particles making up the thermal component are assumed to be noninteracting except that they feel an effective interaction through the external potential and the presence of the condensate. The atoms in the condensate interact through a two-body contact potential modeled in terms of the $s$-wave scattering length. Thus, the approach we take includes interaction effects and we seek to explore the interplay between the nonextensivity and interactions. Similar two-fluid approaches $[15,16]$ are gaining attention because of their simple and intuitive content. Other applications $[17,18]$ employing the two-fluid model have successfully described the two-dimensional systems. Treating the nonextensivity parameter $q$ as an adjustable parameter, we calculate the thermodynamic properties of the system as a function of temperature. We find that there are significant differences in the chemical potential, internal energy, and fraction of atoms in the condensate compared to the standard results brought about by nonextensive statistics.

The rest of the paper is organized as follows. In Sec. II we outline the two-fluid model and approximations to calculate the thermodynamic quantities in an interacting, trapped system of bosons. The effects of nonextensive statistics are incorporated through the nonextensive $\mathrm{BE}$ distribution function. In Sec. III we present our results to highlight the effects of nonextensivity in an interacting system. We conclude with a brief summary.

\section{MODEL AND THEORY}

The ground-state properties of a condensed system of interacting bosons at zero temperature are described by the Gross-Pitaevskii (GP) equation [19]. At finite temperature the interaction of condensed particles with those that are not also have to be taken into account. Within the mean-field theory the condensate is described by the Gross-Pitaevskii equation supplemented by the presence of thermal particles 


$$
\begin{aligned}
- & \frac{\hbar^{2}}{2 m} \nabla^{2} \psi(r)+V_{\mathrm{ext}}(\mathbf{r}) \psi(r)+g \psi^{3}(r)+2 g n_{T}(r) \psi(r) \\
& =\mu \psi(r) .
\end{aligned}
$$

In the above $g=4 \pi \hbar^{2} a / m$ is the coupling constant with $a$ the $s$-wave scattering length, $V_{\text {ext }}(\mathbf{r})=m \omega^{2} r^{2} / 2$ is the harmonic confining potential for isotropic traps, and $n_{T}(r)$ is the density of the thermal particles. $a>0$ signifies short-range repulsive interactions between the atoms. $\mu$ is the chemical potential to be determined from the knowledge of the total number of particles

$$
N=N_{0}+\int d \epsilon \rho(\epsilon)\langle n(\epsilon)\rangle,
$$

where $N_{0}=\int d^{3} r \psi^{2}(r)$ is the number of condensed atoms, $\rho(\epsilon)$ is the density of states, and $\langle n(\epsilon)\rangle$ is the distribution function for bosons. We treat the noncondensed particles as noninteracting bosons in an effective potential [20] $V_{\text {eff }}(r)$ $=V_{\text {ext }}(r)+2 g n_{T}(r)+2 g \psi^{2}(r)$, and calculate the semiclassical density of states using

$$
\rho(\epsilon)=\frac{(2 m)^{3 / 2}}{4 \pi^{2} \hbar^{3}} \int_{V_{\mathrm{eff}}(r)<\epsilon} d^{3} r \sqrt{\epsilon-V_{\mathrm{eff}}(r)} .
$$

Because $V_{\text {eff }}(r)$ depends on $\psi^{2}(r)$ and $n_{T}(r)$ and they in turn depend on $V_{\text {eff }}(r)$, the above set of equations have to be solved self-consistently. Rather than going through a fully numerical solution of the above set of equations we make some simplifying assumptions to treat the interactions perturbatively. Such an approximate scheme [14] works remarkably well to reproduce the fully self-consistent results, thus, we are motivated to employ the simpler approach.

We now introduce the simplifications and approximations to enable us to calculate various thermodynamic properties and density distribution of the condensed and thermal particles in the system perturbatively. First, the Thomas-Fermi (TF) approximation gives

$$
\psi^{2}(r)=\frac{1}{g}\left[\mu-V_{\mathrm{ext}}(r)-2 g n_{T}\right] \theta\left(\mu-V_{\mathrm{ext}}-2 g n_{T}\right),
$$

where $\theta(x)$ is the unit step function. The TF approximation neglects the kinetic energy term in Eq. (1) compared to the interactions and is known to be reliable except for $T$ close to the BEC transition temperature [21]. Next, we consider the situation to zero order in $g n_{T}$ that yields

$$
N_{0}=\left(\frac{2 \mu}{\hbar \omega}\right)^{5 / 2} \frac{a_{\mathrm{HO}}}{15 a},
$$

for the number of condensed particles. The density of states within the same approximation is obtained to be [14]

$$
\begin{aligned}
\rho(\epsilon)= & \frac{1}{\pi(\hbar \omega)^{3}}\left[2 \sqrt{\mu}(\epsilon-\mu)^{3 / 2}+\epsilon^{2} \tan ^{-1} \sqrt{\frac{\epsilon-\mu}{\mu}}\right. \\
& \left.+(2 \mu-\epsilon)^{2} \ln \frac{\sqrt{|\epsilon-2 \mu|}}{\sqrt{\mu}+\sqrt{\epsilon-\mu}}\right]
\end{aligned}
$$

for $\mu>0$, and

$$
\rho(\epsilon)=\frac{\epsilon^{2}}{2(\hbar \omega)^{3}}
$$

for $\mu<0$. Note that the density of states expressions we use include $\mu$ that originates from $V_{\text {eff }}(r)$ [c.f. Eq. (3)]. $\mu$ embodies the interaction effects. More simplified forms of $\rho(\epsilon)$ were used by Salasnich [11] and Fa and Lenzi [12] in their treatment of a noninteracting system.

If we use the standard Bose-Einstein distribution function at temperature $T,\langle n(\epsilon)\rangle=[\exp \beta(\epsilon-\mu)-1]^{-1}$, with $\beta$ $=1 / k_{B} T$, we recover results obtained by Minguzzi et al. [14]. In the nonextensive statistical mechanics of Tsallis, the BE distribution function is given by $[1,4]$

$$
\langle n(\epsilon)\rangle_{q}=\frac{1}{[1+\beta(q-1)(\epsilon-\mu)]^{1 /(q-1)}-1},
$$

where $q$ is a parameter signifying the nonextensivity of the system. As $q \rightarrow 1, \quad\langle n(\epsilon)\rangle_{q}$ goes over to the standard BoseEinstein distribution function. For $q$ close to unity, i.e., the nonextensivity is weak, a Taylor expansion in $(q-1)$ to first order yields

$$
\langle n(\epsilon)\rangle_{q} \approx \frac{1}{e^{\beta(\epsilon-\mu)}-1}+\frac{1}{2}(q-1) \frac{\beta^{2}(\epsilon-\mu)^{2} e^{\beta(\epsilon-\mu)}}{\left[e^{\beta(\epsilon-\mu)}-1\right]^{2}} .
$$

As we have noted, Salasnich [11] used the approximate form of the $\mathrm{BE}$ distribution function in the nonextensive statistics and found a marked difference in the BEC transition temperature compared to the ordinary case. Fa and Lenzi [12] pointed out the importance of using the exact nonextensive BE distribution function by demonstrating deviations as $|q-1|$ becomes large. We, thus, use the full distribution function given in Eq. (8) in our subsequent calculations for an interacting system of bosons.

\section{RESULTS AND DISCUSSION}

We have calculated the thermodynamic properties of a trapped interacting Bose gas in the two-fluid model, by treating the parameter $g n_{T}$ to zero order as set out in the previous section. We take the various physical parameters to be used in the numerical calculation from the experimental work of Ensher et al. [22] on a gas of ${ }^{87} \mathrm{Rb}$ atoms. We take $a / a_{\mathrm{HO}}$ $=0.0062$ for the $s$-wave scattering length describing interaction effects, where $a_{\mathrm{HO}}=(\hbar / m \omega)^{1 / 2}$ is the harmonic oscillator length. For simplicity we assume an isotropic harmonic oscillator well for the trapping potential. In the temperature dependent thermodynamic quantities we use the scale set by 


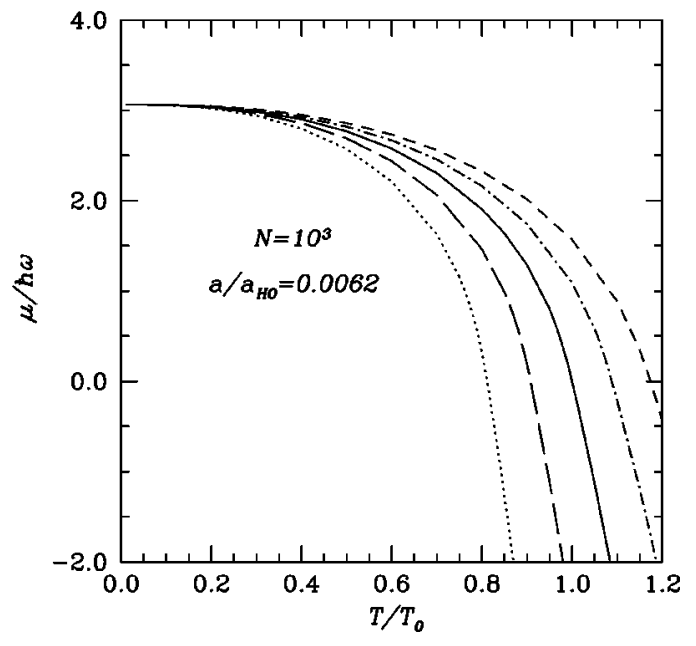

FIG. 1. The chemical potential of the interacting, trapped Bose gas as a function of temperature. The solid line indicates $\mu$ calculated using the standard BE distribution function. The dotted and long dashed lines below the solid line are for nonextensive statistics with $q=1.1$ and $q=1.05$, respectively. The short dashed and dashdotted lines above the solid line are also for nonextensive statistics with $q=0.9$ and $q=0.95$, respectively.

the transition temperature of a noninteracting bosons in a harmonic trap, $k_{B} T_{0}=\hbar \omega(N / \zeta(3))^{1 / 3}$, where $\zeta(3) \approx 1.202$ is the Riemann zeta function.

The chemical potential $\mu$ as calculated from Eq. (2) for a system of $N=10^{3}$ particles and various values of the nonextensivity parameter $q$ is illustrated in Fig. 1. We find that there are significant differences between the $q=1$ (standard BE distribution) and $q \neq 1$ cases especially at higher temperatures $T \sim T_{0}$. At very low temperatures, the nonextensivity does not appear to be important. This is because the change in the form of the excited state populations due to nonextensive statistics [Eq. (8) or (9)] is negligible at low temperatures. For $q<1$, we have used an upper cutoff $\beta$ ( $\epsilon$ $-\mu) \leqslant 1 /(1-q)$ in the nonextensive distribution function $\langle n(\epsilon)\rangle_{q}$. The energy cutoff for $q<1$ naturally arises from the constraints imposed on internal energy as discussed by Tsallis et al. [23]. It has been used in the previous calculations $[11,12]$ on BEC for noninteracting systems. In this case, the chemical potential decreases more rapidly than the $q=1$ case, therefore, the critical temperature $T_{c}$ at which $\mu$ becomes zero is lower than $T_{0}$.

Figure 2 shows the temperature dependence of the condensate fraction $N_{0} / N$ for various values of the nonextensivity parameter $q$. In Fig. 2(a) we concentrate on the $q>1$ case. As a consequence of the behavior of the chemical potential the condensate fraction $N_{0} / N$ decreases more rapidly than the standard BE distribution result. We can clearly see the decreasing of critical temperature $T_{c}$ as a result of nonextensive statistics. Also plotted is the expression for the condensate fraction of a noninteracting Bose gas in a harmonic trap, $N_{0} / N=1-\left(T / T_{0}\right)^{3}$. In Fig. 2(b) we show our results for $q<1$. In this case, the condensate fraction stays above the result using the standard $\mathrm{BE}$ distribution function. Consequently, the BEC transition temperature $T_{c}$ is larger than that for standard BE distribution function.
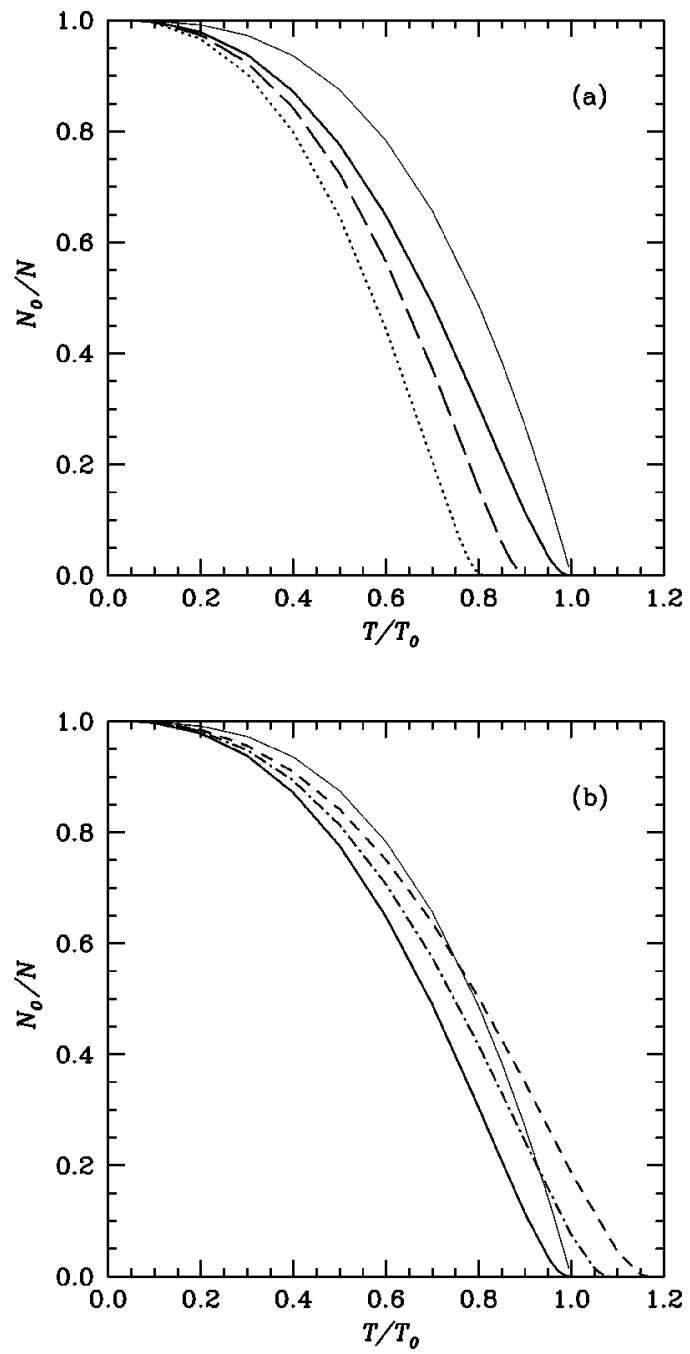

FIG. 2. (a) The fraction of particles in the condensate as a function of temperature. The thin solid line is for a trapped noninteracting system. The thick solid line is $N_{0} / N$ calculated using the standard BE distribution function. The dotted and dashed lines are for nonextensive statistics with $q=1.1$ and $q=1.05$, respectively. (b) The fraction of particles in the condensate as a function of temperature. The thin solid line is for a trapped noninteracting system. The thick solid line is $N_{0} / N$ calculated using the standard BE distribution function. The short dashed and dash-dotted lines are for nonextensive statistics with $q=0.9$ and $q=0.95$, respectively.

The changes on $N_{0} / N$ and $T_{c}$ due to nonextensive statistics follow directly from Eq. (8) or (9). Clearly, for $q>1$, the population of an excited state (for a given $T$ and $\mu$ ) is higher than for the extensive statistics $(q=1)$. Thus, the condensate will vanish more rapidly leading to a lowered $T_{c}$. Similarly, for $q<1$, the population of an excited state is lower than for the $q=1$ case, resulting in an increased $T_{c}$. Our calculations provide quantitative information based on these observations.

Having calculated the temperature dependence of the number of particles in the condensate, we now turn to the density profiles of the condensed and noncondensed particles. The density of condensed atoms is simply given by $n_{0}(r)=\psi(r)^{2}$. For the thermal (noncondensed) particles in 

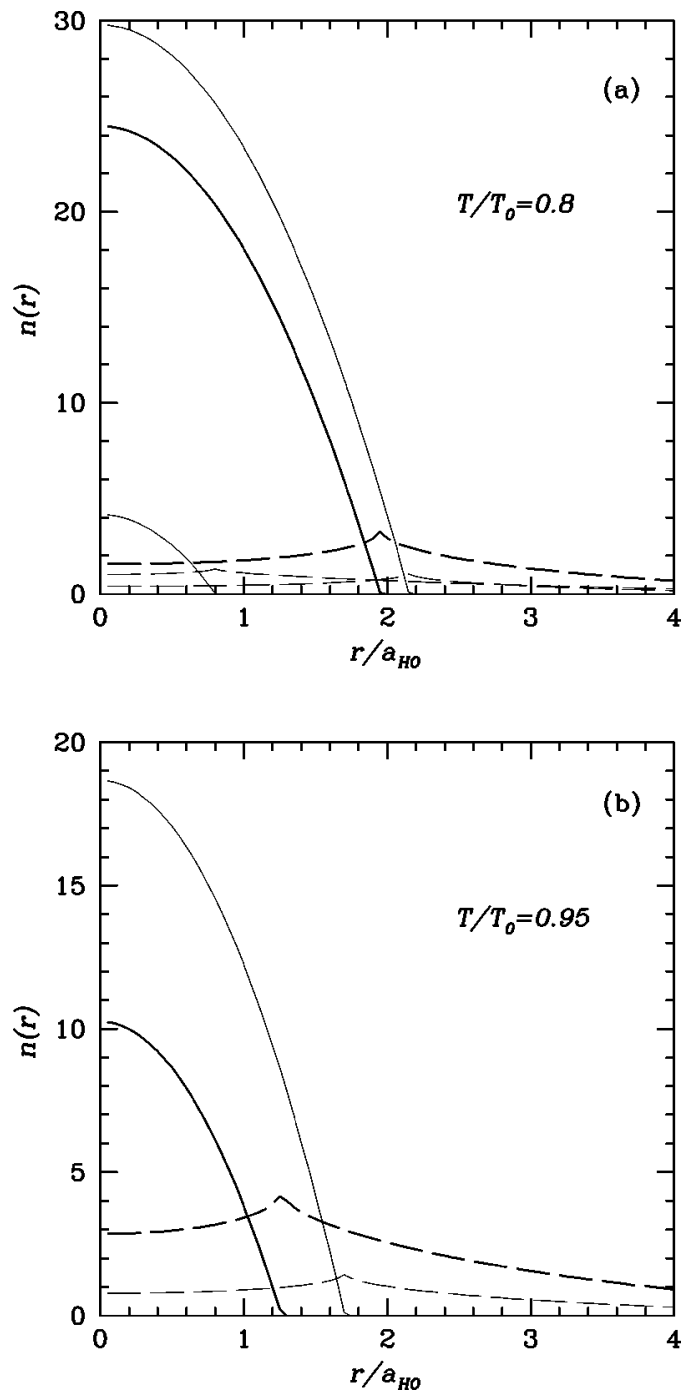

FIG. 3. (a) The density distributions of the condensed $n_{0}(r)$ (solid lines) and noncondensed $n_{T}(r)$ (dashed lines) particles as a function of the radial distance at $T=0.8 T_{0}$. The thick lines correspond to the calculation using the standard BE distribution function. The thin lines are for nonextensive statistics. The smaller condensate and the corresponding thermal cloud is for $q=1.1$, whereas the larger condensate and corresponding thermal cloud is for $q=0.9$. (b) The density distributions of the condensed $n_{0}(r)$ (solid lines) and noncondensed $n_{T}(r)$ (dashed lines) particles as a function of the radial distance at $T=0.95 T_{0}$. The thick lines correspond to the calculation using the standard BE distribution function. The thin lines are for nonextensive statistics with $q=0.9$.

keeping with the noninteracting bosons in an effective potential picture, we use

$$
\begin{aligned}
n_{T}(r)= & \frac{1}{(2 \pi \hbar)^{3}} \int d^{3} p \\
& \times \frac{1}{\left[1+\beta(q-1)\left(p^{2} / 2 m-V_{\mathrm{eff}}(r)-\mu\right)\right]^{1 /(q-1)}-1} .
\end{aligned}
$$

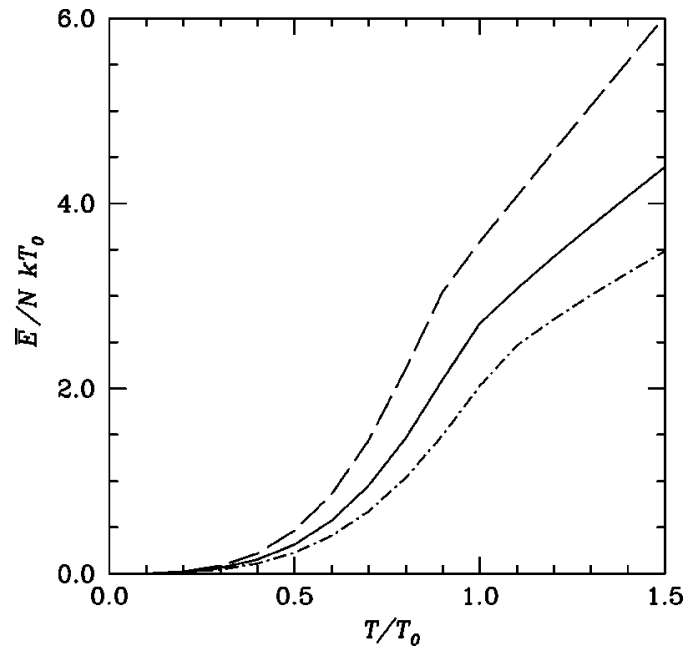

FIG. 4. The average internal energy of a trapped Bose gas per particle as a function of temperature. The solid line indicates $\bar{E}$ calculated with the standard BE distribution function. The dashed and dash-dotted lines are for nonextensive statistics with $q=1.05$ and $q=0.95$, respectively.

Here we use the previously calculated values of $\mu$ and $V_{\text {eff }}(r)$ to obtain $n_{T}(r)$. Our results for the density profiles of condensed and noncondensed particles are depicted in Fig. 3. In Fig. 3(a) we show $n_{0}(r)$ and $n_{T}(r)$ for $q=1.1, \quad q=1$ (Boltzmann-Gibbs statistics), and $q=0.9$ at $T=0.8 T_{0}$ in a system of $N=10^{3}$ particles. For $q=1.1$, the system is close to the BEC transition temperature, therefore, the condensate is rather small. Consequently the thermal component is quite sizeable compared to the condensate. For $q=0.9$, the condensate is larger than that in the standard calculation with ordinary BE distribution function. In all cases, the density distribution of thermal particles $n_{T}(r)$ makes a peak around the region where the condensate vanishes, and it extends out to large distances. In Fig. 3(b) we show the density distributions at $T=0.95 T_{0}$. At this temperature condensate component for $q=1.1$ has already disappeared. The system obeying the usual Boltzmann-Gibbs statistics $(q=1)$ is close to the transition temperature. We plot $n_{0}(r)$ and $n_{T}(r)$ for $q=1$ and $q=0.95$ cases only. When $q<1$, we have used energy or momentum cutoffs mentioned earlier in the calculation of density distributions. From the knowledge of configuration space distributions we can calculate the momentum distributions by Fourier transformation. The examples we have shown indicate measurable differences in the density profiles of condensed and thermal components of the interacting Bose gas due to nonextensive statistics. These quantities are amenable to experimental observations, thus, analysis in terms of nonextensivity should be possible.

We have also calculated the internal energy of the system using

$$
E=\int_{0}^{\infty} d \epsilon \epsilon \rho(\epsilon)\langle n(\epsilon)\rangle_{q}
$$

within the nonextensive statistics. From the knowledge of $E(N, T)$ the specific heat $c_{V}$ and other thermodynamic prop- 
erties of the system can be calculated. Figure 4 displays the energy per particle as a function of temperature. We observe that influence of nonextensive statistics becomes more visible at higher temperatures.

We have reported our calculations of the effects of nonextensivity on the thermodynamics of an interacting trapped system of bosons, treating the nonextensivity parameter $q$ as adjustable. There has been attempts at relating $q$ to the underlying dynamics of the system [24]. It has been argued that the value of $q$ for a specific system is mainly controlled by the range of interactions. From our calculations shown in Fig. 2, it appears that $q>1$ emulates the behavior of interacting system of bosons in a trap potential. This is because experimental results indicate a lowering of $T_{c}$ [22]. These changes in $T_{c}$ have been fully accounted for by repulsive interactions and finite size effects. At the same time, in their variational approach to solve the GP equation at zero temperature within nonextensive statistics, Fa et al. [13] have used $q<1$ for repulsive interactions. It would be desirable to explore the microscopic foundation of the value of $q$ parameter in the present context.

In summary, we have considered a mean field, semiclassical two-fluid description of an interacting trapped Bose system obeying nonextensive statistics. The degree of non- extensivity is characterized by a parameter in the BoseEinstein distribution function. We have calculated the thermodynamic properties, condensate fraction, and density distributions of condensed and thermal particles to compare the differences between nonextensive statistics and the standard one. Our results indicate small deviations from ordinary $\mathrm{BE}$ statistics may result in large changes in the BEC transition temperature for interacting systems. There may be several possible directions for future calculations. The effects of nonextensivity may be further explored in systems of lower dimensionalities, as recent experiments are getting to be performed in two and one-dimensional condensates. Our calculations may easily be extended to account for asymmetric trapping potentials. Finally, the behavior of trapped Fermi gases may also be investigated within nonextensive statistical mechanics.

\section{ACKNOWLEDGMENTS}

This work was supported by the Scientific and Technical Research Council of Turkey (TUBITAK), by NATO, by the Turkish Department of Defense, and by the Turkish Academy of Sciences (TUBA).
[1] C. Tsallis, J. Stat. Phys. 52, 479 (1988); E. M. F. Curado and C. Tsallis, J. Phys. A 24, L69 (1991); 24, 3187(E) (1991); 25, 1019(E) (1992).

[2] M. Buiatti, P. Grigolini, and A. Montagnini, Phys. Rev. Lett. 82, 3383 (1999).

[3] I. Koponen, Phys. Rev. E 55, 7759 (1998).

[4] F. Büyükkiliç, D. Demirhan, and A. Güleç, Phys. Lett. A 197, 209 (1995).

[5] M. R. Ubriaco, Phys. Rev. E 60, 165 (1999).

[6] A. K. Rajagopal, R. S. Mendes, and E. K. Lenzi, Phys. Rev. Lett. 80, 3907 (1998); E. K. Lenzi, R. S. Mendes, and A. K. Rajagopal, Phys. Rev. E 59, 1398 (1999).

[7] D. F. Torres and U. Tirnakli, Physica A 261, 499 (1998).

[8] F. Büyükkiliç and D. Demirhan, Eur. Phys. J. B 14, 705 (2000).

[9] M. H. Anderson, J. R. Ensher, M. R. Matthews, C. E. Wieman, and E. A. Cornell, Science 269, 198 (1995); K. B. Davis, M.-O. Mewes, M. R. Andrews, N. J. van Druten, D. S. Durfee, D. M. Kurn, and W. Ketterle, Phys. Rev. Lett. 75, 3969 (1995); C. C. Bradley, C. A. Sackett, and R. G. Hulet, ibid. 78, 985 (1997).

[10] F. Dalfovo, S. Giorgini, L. Pitaevskii, and S. Stringari, Rev. Mod. Phys. 71, 463 (1999); A. S. Parkins and D. F. Walls, Phys. Rep. 303, 1 (1998); A. J. Leggett, Rev. Mod. Phys. 73, 307 (2001); Ph. W. Courtieille, V. S. Bagnato, and V. I. Yukalov, Laser Phys. 11, 659 (2001).
[11] L. Salasnich, Int. J. Mod. Phys. B 14, 405 (2000); 15, 1253 (2001).

[12] K. S. Fa and E. K. Lenzi, Braz. J. Phys. 31, 317 (2001).

[13] K. S. Fa, R. S. Mendes, P. R. B. Pedreira, and E. K. Lenzi, Physica A 295, 242 (2001).

[14] A. Minguzzi, S. Conti, and M. P. Tosi, J. Phys.: Condens. Matter 9, L33 (1997).

[15] R. J. Dodd, K. Burnett, M. Edwards, and C. W. Clark, Acta Phys. Pol. A 93, 45 (1998); R. J. Dodd, M. Edwards, and C. W. Clark, J. Phys. B 32, 4107 (1999).

[16] M. Naraschewski and D. M. Stamper-Kurn, Phys. Rev. A 58, 2423 (1999).

[17] M. Bayindir and B. Tanatar, Phys. Rev. A 58, 3134 (1998).

[18] J. G. Kim and E. K. Lee, J. Phys. B 32, 5575 (1999).

[19] E. P. Gross, Nuovo Cimento 20, 454 (1961); L. P. Pitaevskii, Zh. Éksp. Teor. Fiz. 40, 646 (1961) [Sov. Phys. JETP 13, 451 (1961)].

[20] V. Bagnato, D. E. Pritchard, and D. Kleppner, Phys. Rev. A 35, 4354 (1987).

[21] F. Dalfovo and S. Stringari, Phys. Rev. A 53, 2477 (1996).

[22] J. R. Ensher, D. S. Jin, M. R. Matthews, C. E. Wieman, and E. A. Cornell, Phys. Rev. Lett. 77, 4984 (1996).

[23] C. Tsallis, R. S. Mendes, and A. R. Plastino, Physica A 281, 534 (1998).

[24] M. L. Lyra and C. Tsallis, Phys. Rev. Lett. 80, 53 (1998); C. Anteneodo and C. Tsallis, ibid. 80, 5313 (1998). 